ALICIA

MORALES-PEREYRA

CARLOS

JIMÉNEZ-MARTÍNEZ
Investigadora Predoctoral del Departamento de Bellas Artes. Universidad de La Laguna amoralep@ull.edu.es

Profesor Contratado Doctor del Departamento de Bellas. Universidad de La Laguna carjimen@ull.edu.es

\title{
Codiseño y dinamización cultural comunitaria. Reflexiones conceptuales, metodológicas y prácticas a partir de una experiencia desde la periferia
}




\section{CO-DESIGN AND COMMUNITY CULTURAL DYNAMIZATION. CONCEPTUAL, METHODOLOGICAL AND PRACTICAL REFLECTIONS FROM AN EXPERIENCE FROM THE PERIPHERY}

\section{ABSTRACT}

We analyze the potential to generate critical collaborative and pedagogical practices from Design, which contribute to processes of community artistic and cultural development in contexts of marginal, informal and peripheral urbanism with respect to production centers. We start from the experience of joint work since 2016 between a faculty of fine arts and a nearby self-construction neighborhood; Las Moraditas de Taco, in Santa Cruz de Tenerife. The evidence that "professors are fed up with the classrooms spaces and students lack more experiences in the streets" joins the neighborhood claim; "we live on a mountain but nobody sees us", to explore models of living labs for creation and service-learning, in the recovery and valorization of collective memory, intangible cultural heritage, sense of belonging, dignity and beautification of public space or the external projection of positive values.

Special attention is paid to designers' emerging roles and competencies, such as facilitator, catalyst or mediator. We highlight the relevance of adopting methodological frameworks based on participatory action-research, thus reorienting the traditional, institutional and topdown culture project, towards iterative process cultures of citizen innovation, from which to generate, stimulate and root transformations within the community and the territory.

\section{Keywords}

Co-design; community dynamization; intangible cultural heritage; living labs; participatory methodologies

\section{RESUMEN}

Analizamos el potencial para generar prácticas colaborativas y pedagógicas críticas desde el Diseño, que contribuyan a procesos de desarrollo artístico y cultural comunitario en contextos de urbanismo marginal, informal y periféricos respecto a los centros de producción. Partimos de la experiencia de trabajo conjunto desde 2016 entre una facultad de bellas artes y un barrio de autoconstrucción cercano; Las Moraditas de Taco, en Santa Cruz de Tenerife. La evidencia de que "al profesorado le sobra aula y al alumnado le falta calle" se une a la reivindicación vecinal de "vivimos en una montaña pero nadie nos ve" para explorar modelos de laboratorios vivos de creación y aprendizaje-servicio, en la recuperación y valorización de la memoria colectiva, el patrimonio cultural inmaterial, el sentido de pertenencia, la dignificación y embellecimiento del espacio público o la proyección exterior de valores positivos.

Prestamos especial atención a la manifestación de roles y competencias emergentes del diseñador, como facilitador, catalizador o mediador. Constatamos la pertinencia de adoptar marcos metodológicos de investigación-acción participativa, que reorienten la tradicional cultura del proyecto institucional de arriba-abajo hacia una cultura de procesos iterativos de innovación ciudadana desde los que generar, dinamizar y arraigar las transformaciones en la comunidad y el territorio.

\section{Palabras clave}

Codiseño; dinamización comunitaria; patrimonio cultural inmaterial; laboratorios vivos; metodologías participativas 


\section{INTRODUCCIÓN}

El espacio público es la esfera donde las diferencias sociales se diluyen a través de la construcción y apropiación de las zonas de uso colectivo. Este facilita el encuentro y el diálogo, además de otras múltiples posibilidades sin limitar su uso de tránsito. Sin embargo, según Torres-Tovar (2019), los territorios y sus lugares se encuentran cada vez más fragmentados a causa de la privatización. Es por ello, que el espacio público debe trascender de la idea de zona residual de la propiedad privada, reducido casi únicamente al tránsito de peatones y vehículos. Hablamos de la reapropiación de estos lugares para el disfrute colectivo ciudadano, permitiendo la articulación de tejidos urbanos y facilitando el encuentro, intercambio e interrelación entre los representantes del territorio.

La configuración de una ciudad incluyente solo es posible mediante la participación de varios agentes (Torres-Tovar, 2019). En un contexto donde la comunidad se encuentra en continua construcción colectiva del espacio público, los diseñadores poseen un papel fundamental para visualizar lo que pasa desapercibido, acercando al territorio con sus habitantes (Pilay y Neves, 2020). Este artículo pretende profundizar en la apropiación, acondicionamiento y dinamización de los espacios públicos de encuentro a través del diseño y la intervención comunitaria. Con este fin, nos apoyaremos en el caso de la iniciativa Amoraditas en el barrio de Las Moraditas.

\subsection{Contexto territorial: Las Moraditas, lecciones de un barrio periférico de autoconstrucción}

En su libro Diseño de la Periferia. Debates y experiencias, Gui Bonsiepe (1985) entiende el diseño en su sentido más amplio, más allá del micronivel de la cultura material, haciéndolo emerger como una forma de cultura emancipatoria y con una finalidad cultural. Así, esta disciplina es entendida como una actividad tecnológica y social que se integra en la cotidianidad, centrándose en la calidad funcional más allá de cuestiones estéticas.

En las zonas más turistificables, los diseñadores son vistos como agentes vitales para la determinación del éxito de estos territorios. Sin embargo, el potencial del diseño en las zonas periféricas queda desaprovechado. Consecuentemente, el diseño de la periferia debe desvincularse del centro, cambiando su interdependencia, socializando con las instituciones pertinentes y posicionando a sus profesionales como agentes capaces de catalizar sus necesidades y traducirlas en mejoras.

Canarias pertenece al grupo de nueve regiones adscritas a tres estados miembros de la Unión Europea denominadas "Regiones Ultra Periféricas" (RUP). Esta designación se les atribuye al separarse entre sí por miles de kilómetros, ubicándose incluso en mares y océanos distintos al resto del territorio al que pertenecen. Si bien comparten características geográficas, climáticas, de biodiversidad y socioeconómicas, se diferencian por diversas particularidades con el resto del territorio, ya sea por su insularidad, aislamiento, orografía o dimensión.

Esta investigación se ubica en el archipiélago canario, en la isla de Tenerife y específicamente en la entidad de población de Taco, en uno de sus dos barrios pertenecientes a la municipalidad de Santa Cruz de Tenerife: Las Moraditas (Figura 1). 

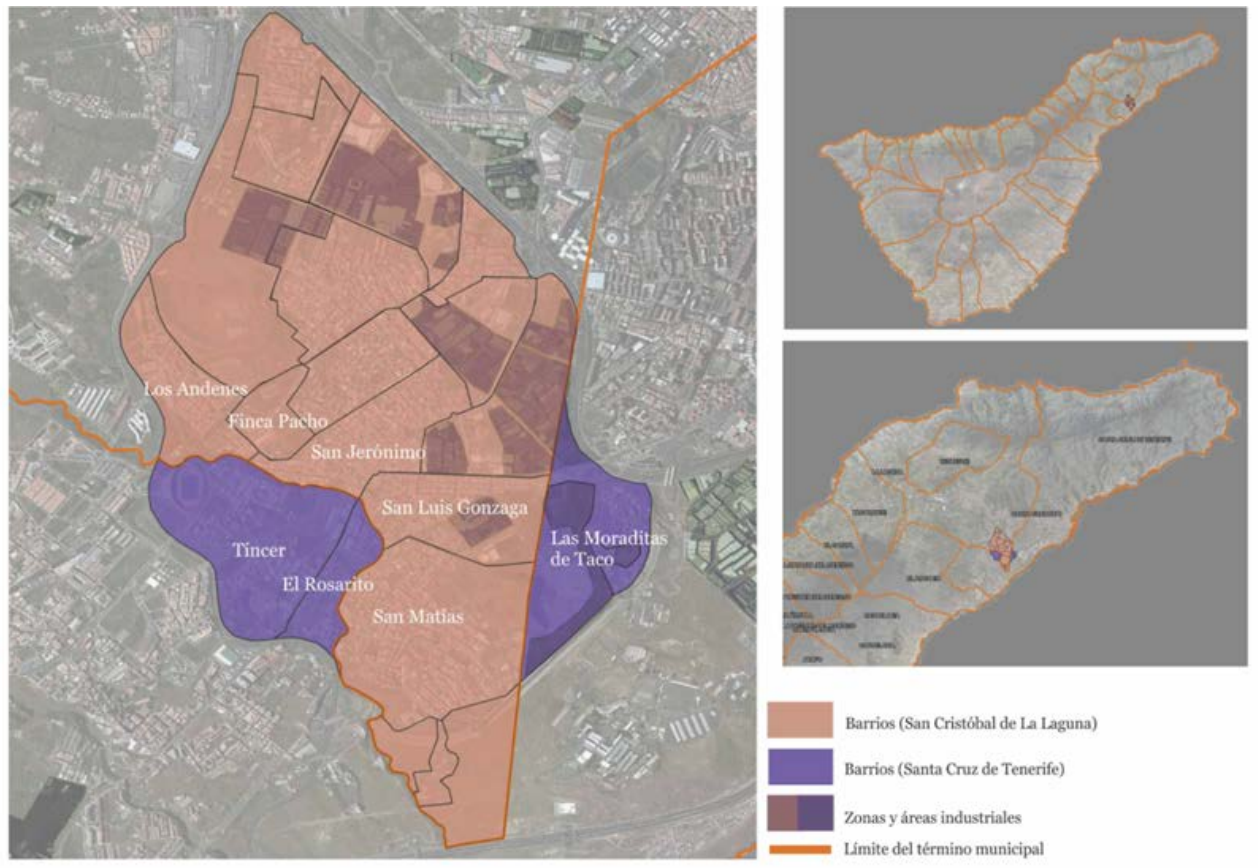

Figura 1. Mapa de Taco contextualizado en la isla de Tenerife. Fuente: ICI-Taco, 2015.

Configurada mediante un proceso espontáneo e ilegal, esta comunidad periférica es un ejemplo de aquellos asentamientos conformados por viviendas de autoconstrucción. Estas edificaciones, construidas con técnicas y materiales muy primarios, son modeladas al margen del planeamiento urbanístico, sobre suelo rústico no preparado para su utilización ciudadana.

Las Moraditas, a pesar de encontrarse a los bordes de la ciudad central, posee una ubicación geográfica privilegiada, pues se asienta en la vertiente expuesta a Santa Cruz de la Montaña de Taco. Así, se encuentra en una zona estratégica elevada al tránsito, próxima a la Autopista del Norte de Tenerife o TF-5, que recorre el nordeste y norte de la isla, enlazando con importantes núcleos urbanos como Santa Cruz, San Cristóbal de La Laguna o Tacoronte.

Taco, en su contexto más amplio, se trata de un emplazamiento comercial e industrial que tradicionalmente se asociaba a la marginalidad y el conflicto. Sin embargo, esta imagen externa se ha visto renovada gracias al trabajo de múltiples grupos que han conseguido mostrar su verdadera identidad multicultural; y a la intervención de la administración, mejorando su conexión con el resto de la conurbación mediante la adecuación de carreteras y la proximidad de la autopista y diversas líneas de autobús y tranvía.

Se trata de un territorio con una dinámica migratoria relevante, respondiendo especialmente al importante movimiento migratorio interno ocurrido durante la segunda mitad del siglo $\mathrm{XX}$, donde inmigrantes tanto de Tenerife como de las islas no capitalinas, especialmente de La Gomera, migraron del campo a la ciudad en busca de nuevas oportunidades laborales. Estas nuevas actividades urbanas surgen tras la expansión del área portuaria, el desarrollo de infraestructuras de comunicación o incluso, el intento de preservar la actividad agrícola (Zapata, Mesa y Fonte, 2020, p.59-61). 
En definitiva, Taco y concretamente el barrio de Las Moraditas, resultan un claro ejemplo de la formación de la periferia urbana de Santa Cruz de Tenerife, al tiempo que ilustra uno de los procesos más destacados de la Geografía Urbana contemporánea y sus consecuentes efectos socioespaciales.

\section{AMORADITAS: APROXIMACIÓN A LA INICIATIVA Y SU CONTEXTO}

Ante las necesidades actuales percibidas de este territorio surge Amoraditas (Figura 2). Esta iniciativa sin ánimo de lucro ni financiación externa, contribuye a la recuperación de la memoria colectiva y la dignificación del barrio de Las Moraditas a través del arte, el diseño y la creación en comunidad.

Una de las funciones sociales del arte es la de dar una imagen precisa y definir los rasgos de un cuadro social difuso. Uno de los aspectos más destacados de la vida postmoderna es la erradicación de la historia y la pérdida de la memoria colectiva. [...] Una ciudad es un conjunto de procesos históricos en continuo desarrollo, lo que es lo mismo que decir que la ciudad encarna y protagoniza una historia. (Blanco, Carrillo, Claramonte y Expósito, 2001, p.176).

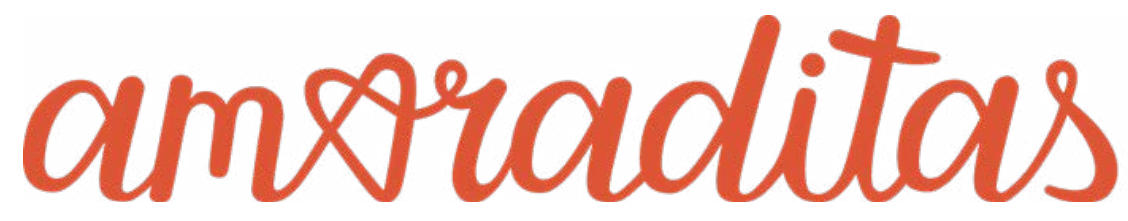

Figura 2. Logotipo de la iniciativa Amoraditas. Fuente: Alba Benítez, 2020.

De este modo, Amoraditas procura colaborar en la prevención de la desaparición de la historia oral de Las Moraditas, pues apenas había sido registrada con anterioridad. Paralelamente aborda, de forma táctica, la adecuación y mejora de un territorio institucionalmente olvidado. Convirtiendo así los muros, calles y escaleras del barrio -a través de murales o paneles interpretativos- en los propios narradores de su memoria colectiva.

Este proceso nace de la Semana del Diseño de Tenerife 2016 (Tenerife Design Week, TDW), concretamente del programa de aprendizaje y ejecución de proyectos-retos que coorganiza junto al Cabildo de Tenerife: el Living Design Lab. Este "laboratorio vivo" planteó un debate abierto en torno a diseño cívico, activismo gráfico, diseño social o arquitecturas participativas en la que participó alumnado y profesorado de todas las universidades y entidades vinculadas al diseño de Tenerife. Su programa se divide en 3 grupos: (1) "intervención urbana y textil", (2) "construcciones colectivas" y (3) "gráfica en acción", siendo específicamente este último el que intervino en Las Moraditas. Desde la Facultad de Bellas Artes de Universidad de La Laguna continuamos su labor, adoptando un marco metodológico de investigación-acción participativa, pasando de una "cultura del proyecto" con inicio y final definidos y acotados en el tiempo, a una "cultura del proceso", donde el briefing y las acciones van emergiendo a través del respeto a los ritmos de la comunidad en espacios temporales amplios y con la implicación de múltiples actores sociales. 
Desde entonces, Amoraditas inicia su proceso comunitario desde el diseño en el contexto más amplio del proyecto de Intervención Comunitaria Intercultural, ICl-Taco, el cual finalizó el pasado diciembre de 2020. El proyecto ICI de la Fundación la Caixa, iniciado en 2010, se desarrolló exitosamente en 36 territorios españoles con alta variedad cultural -uno de ellos Taco- abordando la diversidad local con el fin de fomentar la interacción, la convivencia y la cohesión social.

Actualmente, la iniciativa es enteramente promovida por alumnado y profesorado de la Facultad de Bellas Artes de la Universidad de La Laguna, conjuntamente con vecinas, vecinos y amigos del barrio, aunque es un proceso abierto al que todas las personas están invitadas a sumarse. Cada año, durante el curso académico, visitamos a la comunidad en numerosas ocasiones con el alumnado de diferentes asignaturas, para relacionar lo que aprenden en el aula con la realidad de la calle, proponiendo acciones al servicio de la comunidad (véase Figura 3). Apostamos por la vinculación de la academia al territorio como modelo de retroalimentación y aprendizaje, siguiendo la metodología del Aprendizaje-Servicio (ApS) como medio para promover la participación activa de los estudiantes en experiencias participativas que se integran en el currículum académico.

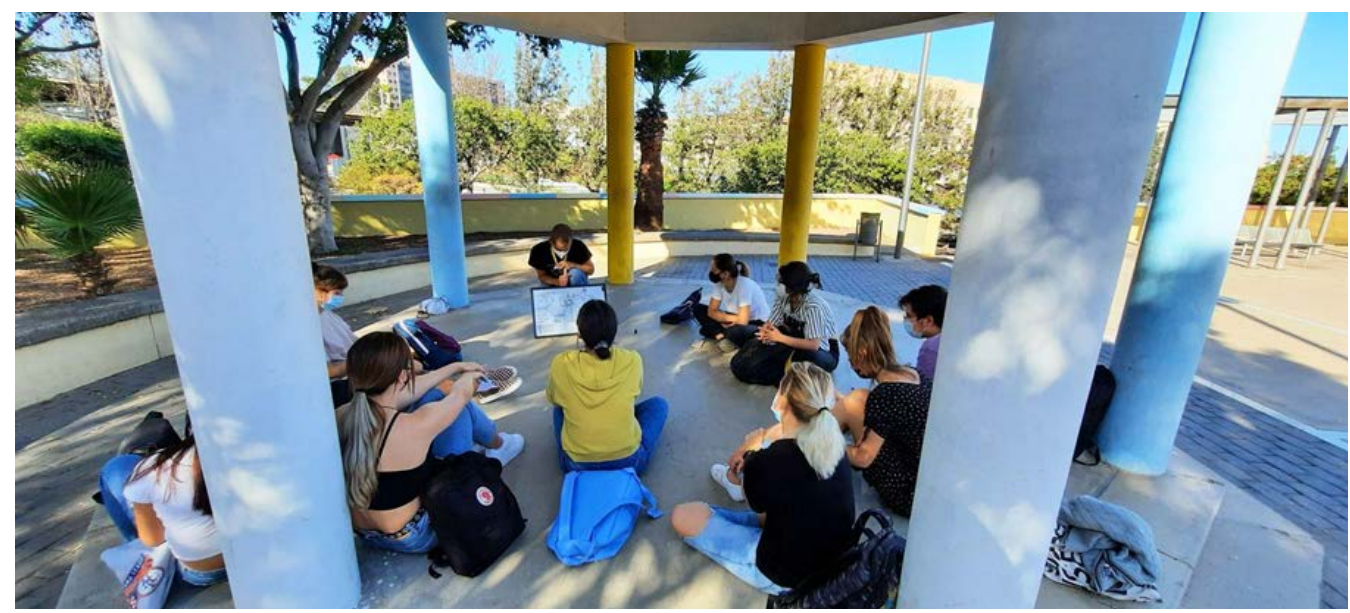

Figura 3. Barrio de Las Moraditas como aula abierta universitaria. Fuente: Elaboración propia, 2020.

Amoraditas trabaja desde una perspectiva transdisciplinar, colaborativa y experimental, desarrollando acciones en ámbitos como la innovación urbana, la participación ciudadana, el placemaking y la cultura colaborativa. Desde la iniciativa, diseñamos, coordinamos e implementamos acciones a escala urbana tratando de incidir en el bienestar social de los habitantes del barrio de Las Moraditas.

Cooperamos con las entidades e instituciones locales con el fin de extraer soluciones integrales de diseño y fomentar una construcción autopromovida de la ciudadanía, potenciando su mejora y su desarrollo. Siguiendo la metodología de "cultura de proceso", se hace especial hincapié en el acompañamiento, identificación de necesidades y creaciones de acciones conjuntas con la ciudadanía, respetando sus ritmos y conjugando sus saberes con la colaboración de expertos que desde sus distintas disciplinas puedan ayudar a hallar soluciones más ricas. 
Creemos que la intervención del diseño a escala territorial ha de trascender la tradicional concepción de cultura de proyecto hacia la cultura del proceso, donde se produzcan situaciones que lleven a cambios significativos. Entendiendo, como ya enunciaba el diseñador Bruce Mau (1998), que "cuando el resultado conduce al proceso solo llegaremos a donde ya hemos estado. Si el proceso conduce al resultado, puede que no sepamos dónde vamos, pero sabremos a dónde queremos llegar".

Hablamos de un proceso iterativo de aprendizaje en el que las sucesivas experiencias, muchas desarrolladas mediante ensayo-error, se suman construyendo y enriqueciendo la iniciativa. Se trata de un proceso dilatado en el tiempo, que se ajusta no solo a los ritmos de la comunidad sino a los académicos, permitiéndonos tener una visión más global de los fenómenos estudiados mediante el continuo testeo y reformulación de las hipótesis. De este modo, los objetivos que persigue la iniciativa son:

- recopilar la memoria oral de Las Moraditas y devolverla a través del diseño gráfico, de servicios y de productos;

- incidir en el sentido de pertenencia de la comunidad potenciando la autoorganización y gestión de los vecinos y vecinas a través de su capacitación y empoderamiento;

- generar espacios y tiempos de encuentro que permitan a la ciudadanía, y demás agentes implicados, dialogar y potenciar las relaciones comunitarias;

- equipar estos espacios comunitarios de forma participativa para fomentar el encuentro vecinal y el acondicionamiento y embellecimiento del barrio;

- incluir al alumnado universitario en procesos participativos a través de las asignaturas para acercarles a la realidad social de su entorno y promover la creación de "proyectos reales".

En definitiva, Amoraditas proporciona un marco de trabajo conceptual y conocimiento situado que mediante el enfoque Investigación Acción Participativa (IAP), permite el diálogo entre un caso de estudio a mostrar y la triangulación de autores. Desde el punto de vista del investigador en diseño, los procesos desarrollados para alcanzar estos objetivos se hacen mediante la devolución y socialización de la memoria gráfica y textual recopilada a través de:

- gráfico-visuales, como la configuración de murales y bancos de imágenes (Figura 4);

- productos-espacios, como dinámicas lúdicas que permitan mostrar historias mediante el juego (Figura 5);

- o servicios, como encuentros y eventos donde compartir el trabajo realizado y realizar aportaciones mediante la participación ciudadana (Figura 6). 


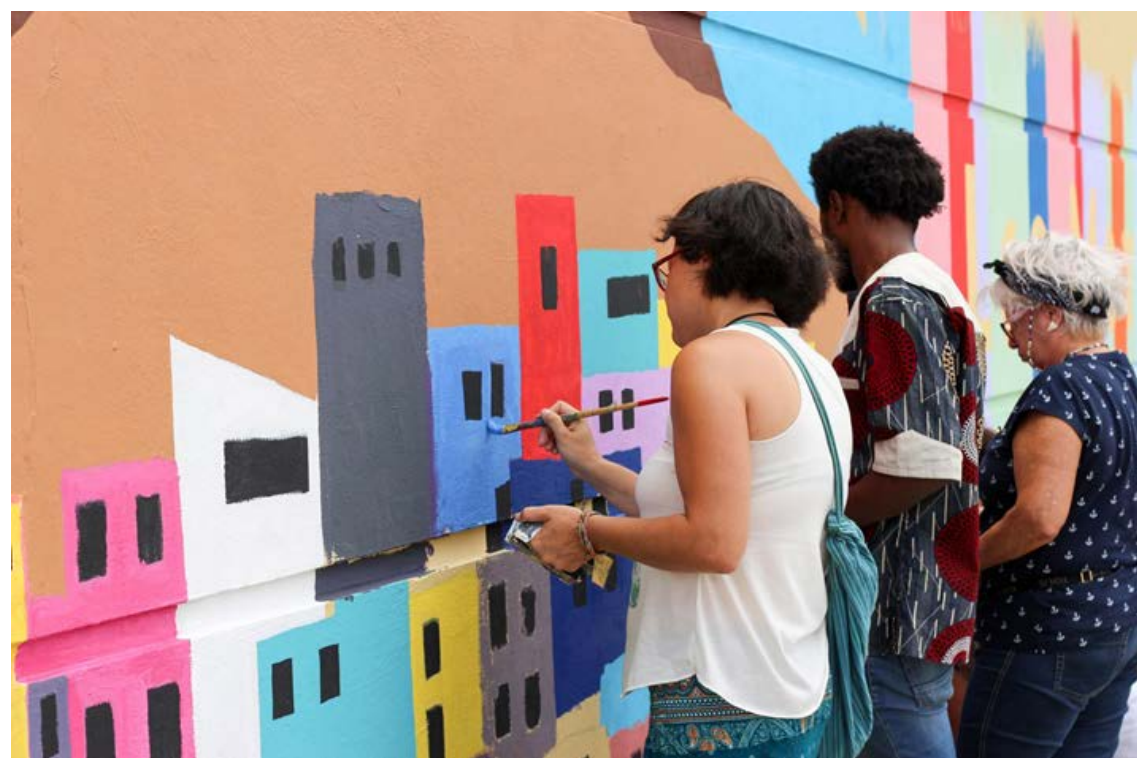

Figura 4. Realización de mural comunitario en La Plaza en el contexto del Encuentro Amoraditas 2018. Fuente: Lorena Oval, 2018.

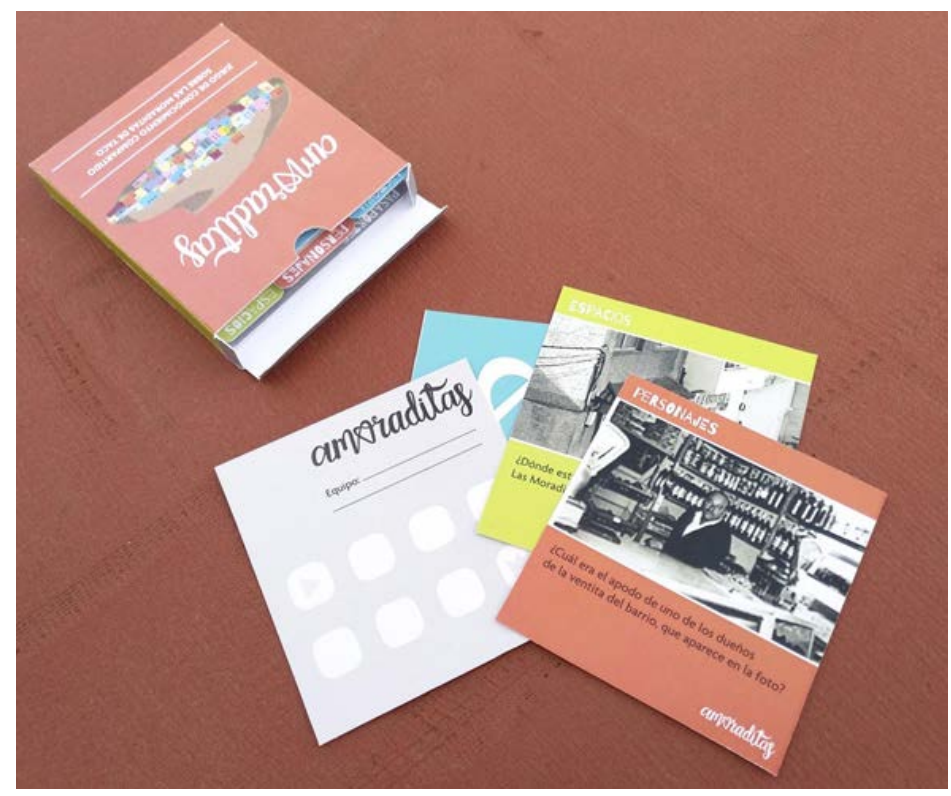

Figura 5. Juego de conocimiento compartido de la memoria colectiva del barrio. Fuente: Elaboración propia, 2018. 


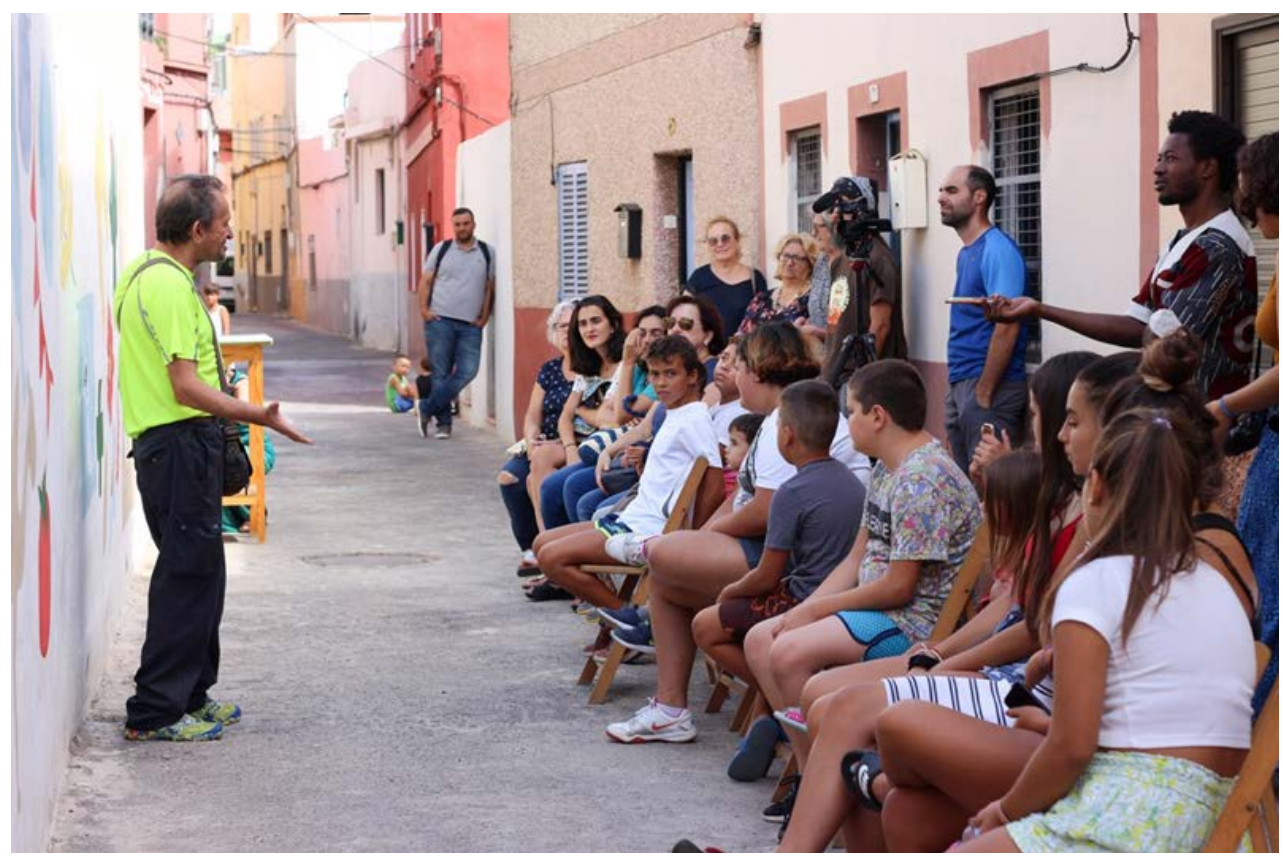

Figura 6. Vecino de Las Moraditas compartiendo con los jóvenes juegos tradicionales e historias populares. Fuente: Lorena Oval, 2018.

\section{1}

\section{Enfoque y marco de acción}

Desde la perspectiva de Ezio Manzini, el diseño como investigación debe entenderse más allá de la propia relación entre investigación y diseño, también desde su poder como actividad capaz de producir conocimiento útil para aquellos que diseñan. Actualmente, en un mundo conectado, los conocimientos de siempre, acumulados en el saber de los expertos en diseño ${ }^{1}$, no resultan suficientes, por lo debe producirse una "investigación constructiva": investigación en diseño cuyos conocimientos sean explícitos, discutibles, transferibles y acumulables, para que puedan servir como punto de partida para otros investigadores (Manzini, 2015, p.49).

Partimos desde un enfoque de Investigación Acción Participativa (IAP), método que implica que investigadores y participantes trabajen colectivamente en busca de soluciones ricas e integradoras. La IAP mejora la enunciación de problemas, la formulación de hipótesis, la obtención de datos, la síntesis y la aplicación (Greenwood, Whyte and Harkavy, 1993, p.177), incitando a que los participantes tomen autonomía e identifiquen sus problemáticas para tomar acción, buscando el cambio social, la democracia y la resolución de retos (Participatory Research Cluster, Institute of Development Studies,n.d.). Se trata de un proceso organizacional de aprendizaje en curso, un enfoque de investigación que promueve el co-aprendizaje, la participación y la transformación organizacional (Greenwood, Whyte and Harkavy, 1993, p.177).

Tratamos de incidir en la comunidad y en su bienestar social generando ideas innovadoras para sus problemáticas sociales. Para ello situamos a las personas en el centro de todo el proceso, siguiendo una aproximación de diseño centrado en las personas (DCP). Esta metodología 
y filosofía busca la realización de productos o servicios útiles y usables que satisfagan las necesidades, deseos y limitaciones de los participantes (Design Toolkit, s.f.).

El "desarrollo comunitario" busca potenciar el tejido social de la comunidad mediante la mejora de sus condiciones de vida, respaldando y priorizando sus propias capacidades. Este programa de trabajo organizado, favorece que los procesos no se agoten, poseyendo una duración determinada, ya que es la propia comunidad quien consensua y prioriza los elementos de mejora a trabajar. (Jiménez-Martínez, 2015, p.39).

Con el objetivo de investigar y desarrollar acciones desde el diseño y la intervención comunitaria, bebemos de múltiples disciplinas con numerosos puntos en común y modos de hacer análogos como el codiseño, el diseño participativo, el diseño cívico o el placemaking.

\subsection{Codiseño}

"El codiseño utiliza un conjunto de métodos y técnicas participativas y se utiliza como un término paraguas para hablar de cocreación, diseño participativo y diseño abierto" (Design Toolkit, s.f.). La idea de codiseño se basa en la colaboración de múltiples y diversos agentes en el proceso de diseño con el fin de solventar problemas complejos. A pesar de ser un término relativamente reciente, se trata de una práctica desarrollada desde hace casi 40 años bajo el nombre de diseño participativo. El codiseño es por tanto un nexo entre dos aproximaciones: (1) el diseño centrado en el usuario, donde éste es estudiado y observado considerándose como "objeto de estudio" y (2) el enfoque participativo, donde los usuarios son estimados como "expertos de su propia experiencia", evidenciando el rol fundamental que poseen desde la idea hasta el desarrollo de la propuesta. (Meroni, Selloni y Rossi, 2018, p.18-19).

"Parte de la premisa de que el diseño es una actividad inherentemente humana (no solo de los diseñadores) y, por tanto, es una actividad social y situada" (Design Toolkit, s.f.). Volviendo al enfoque participativo descrito por Meroni, Selloni y Rossi (2018), en el codiseño se entiende que el diseño vive en el mundo a través de objetos, productos o servicios y, por tanto, las personas al utilizarlos se convierten en expertos de cada dominio. Si el diseñador experto que enuncia Manzini asume el rol de facilitador, las personas pueden convertirse en diseñadores y resolver sus problemas. Consecuentemente hablamos de una democratización del proceso de diseño, "puesto que empodera a las personas y favorece que tomen el control de los productos y servicios que utilizan, y de este modo impacta en su vida, el medio ambiente, la economía y la cultura material" (Design Toolkit, s.f.).

Esta investigación aborda el codiseño y el diseño participativo como un proceso de pensamiento creativo capaz de innovar en el ámbito social gracias a su capacidad de empoderar a los participantes al involucrarles en todo el proceso de diseño, potenciando la conceptualización de ideas y su creatividad en un entorno colaborativo. Estas vertientes han ocasionado una nueva cultura crítica del diseño, valorando el saber y la actividad local, popular y comunitaria, y su aprendizaje intrínseco, como un modelo alternativo de diseño (Garcés, 2020, p.48-49). 
El Diseño Cívico según Domenico Di Siena (2016) puede entenderse como un contexto expandido que nace de la interrelación de conocimientos y prácticas desarrolladas en el ámbito de múltiples disciplinas, entre ellas el diseño social, el placemaking o diseño centrado en el usuario. Se trata de una dinámica que propone llegar a soluciones colectivas, a través de procesos y métodos ideados para el bien de una comunidad, posibilitando la colaboración entre personas y agentes de un territorio en específico.

Tiene en cuenta la dimensión híbrida del territorio, su espacio físico y su esfera digital, asimismo que su relación con su entorno más próximo sin olvidar su conexión o dependencia con el ámbito global, es decir dimensión glocal (Di Siena, 30 de junio de 2015).

Atendiendo a algunas de sus características descritas por Di Siena y Pita (2016) cabe destacar que el diseño cívico forma a diseñadores como facilitadores y conectores transversales capaces de leer, filtrar y gestionar la información territorial, creando oportunidades y desarrollando soluciones desde procesos de inteligencia colectiva. Así, se entiende la educación como factor clave, considerándose como un proceso creativo y colectivo situado, más allá de la transferencia de conocimiento, promoviendo una memoria viva alrededor de cada proyecto. Su ámbito de trabajo defiende el valor del capital humano y creativo, implicando en su proceso necesariamente a las diferentes generaciones y comunidades que habitan en un territorio.

Los diseñadores cívicos son, por tanto, uno de esos diversos expertos en diseño que desarrollan proyectos a escala local, o en otras palabras "diseño como creación de lugares" o placemaking. Agentes "interesados en la regeneración de "lo local" mediante una nueva ecología de los lugares: un ecosistema en el que la cultura y la producción sean capaces de vivir y regenerarse en una relación entre lo local y lo global” (Manzini, 2015, p.58).

La práctica de placemaking inspira a las personas a reimaginar y reinventar colectivamente los espacios públicos, identificando nuevas potencialidades y usos. Se trata de un proceso colaborativo que promueve la configuración de los espacios compartidos en el territorio, favoreciendo las conexiones entre personas que viven en una misma comunidad y aumentando su valor compartido. El placemaking no es una idea, sino una aproximación que presta especial atención a la identidad física, cultural y social que define cada territorio, fomentando su evolución continua (Project for Public Spaces, 2017). Este enfoque multifacético para la planificación, diseño y gestión de los espacios públicos, saca provecho de los activos de una comunidad local, poniendo en valor sus necesidades y deseos para realizar un plan de acción. Enseña a mirar con nuevos ojos y construir una visión colectiva que vea a un lugar desde su totalidad y no atendiendo a fragmentos concretos o aislados. (Barrientos, 2012).

Este estudio se acerca a la innovación cívica mediante el desarrollo de contextos, dispositivos y herramientas para la participación y la inteligencia colectiva. La intervención en el territorio se lleva a cabo desde un enfoque de diseño cívico y placemaking a través de procesos colectivos y participados a escala comunitaria y urbana. 


\title{
3 DISEÑO, PATRIMONIO Y COMUNIDAD
}

Se entiende por patrimonio a todos aquellos elementos identitarios que forman parte de nuestra herencia, materializando nuestra cultura (Ministerio de Educación y Formación Profesional, s.f., p.4).

Si bien, generalmente se asocia a los bienes materiales, también llamados activos "duros" o patrimonio tangible, también incluye los bienes inmateriales, intangibles o activos "blandos" (García, 2021, p.142). Atendiendo a la definición de la UNESCO:

\begin{abstract}
El patrimonio cultural no se limita a monumentos y colecciones de objetos, sino que comprende también tradiciones o expresiones vivas heredadas de nuestros antepasados y transmitidas a nuestros descendientes, como tradiciones orales, artes del espectáculo, usos sociales, rituales, actos festivos, conocimientos y prácticas relativos a la naturaleza y el universo, y saberes y técnicas vinculados a la artesanía tradicional. (Organización de las Naciones Unidas para la Educación, la Ciencia y la Cultura [UNESCO], s.f.).
\end{abstract}

Profundizando en la importancia del patrimonio cultural inmaterial, cabe señalar su relevancia en torno al mantenimiento de la diversidad cultural frente a la globalización. Su comprensión fomenta el diálogo intercultural, el respeto y su transmisión generacional. (UNESCO, s.f.)

Actualmente, al hablar de patrimonio, ya sea de naturaleza material o inmaterial, su vinculación con la participación ciudadana, y la involucración de la comunidad en diferentes proyectos, resulta clave para su conservación a corto y largo plazo (García, 2021, p.142). Haciendo patrimonios comunes podemos generar, impulsar y consolidar el sentimiento colectivo, el compromiso con nuestra comunidad y con nuestro territorio, y buscar las herramientas que permitan cambiar las formas de relacionarnos con nuestra cultura. Se produce así una emancipación del conocimiento mediante la expansión de los saberes y la co-creación de contenidos y metodologías. Asimismo, contribuye a la mejora de la gestión de los elementos patrimoniales o de memoria colectiva y establecer vínculos más fuertes y reales con la población y contexto en el que se encuentran (Ministerio de Educación y Formación Profesional, s.f., p.4).

\subsection{Intervenciones ciudadanas y arte urbano}

El arte urbano, ya sea participativo o no, forma parte del patrimonio tangible, al poder verse, tocarse y sentirse; pero también al intangible, ya que habitualmente se vincula a historias y memorias relacionadas con los espacios (García, 2021, p.142). Este, surgido en busca de la libertad de expresión y el embellecimiento, permite resignificar la ciudad dotándola de personalidad mediante su color, formas y mensajes, evocando a los transeúntes y vecinos a convivir y sentir (Díaz, 2021, p.153). En la calle, nuestro espacio compartido, todo es posible, por lo que lo que ella sucede es susceptible a impregnar en la cultura popular, integrándose en la memoria de la ciudad (García y Luque, 2021, p.106).

Existe un debate abierto en torno a la consideración del arte urbano como patrimonio. Sin embargo, este cumple las condiciones del arte líquido propuesto por Bauman, puesto que se centra en sucesos efímeros de gran impacto social. Concebir el arte como acontecimiento y no como obra, donde lo relevante deja de ser el soporte o su duración, sino precisamente su 
carácter perecedero, el momento de su creación y su procedencia social y política. (García, 2021, p.156-157). Hablamos de un objeto de crítica social y poder, puesto que en ocasiones trasciende la barrera del embellecimiento para darle voz a colectivos vulnerables o como medio de transparentar acciones del gobierno (Díaz, 2021, p.153). Su subalternidad, posible anonimato, pertenencia al espacio público, la creación colectiva y su fugacidad (García, 2021, p.157) lo convierten en "un producto final híbrido, con valores inmateriales y materiales, que en cualquier caso se verá revestidos de temporalidad, casualidad y oportunidad" (García y Luque, 2021, p.106).

Consecuentemente, para analizar la importancia de este tipo de intervenciones en la sociedad es necesario examinarlas no únicamente desde un punto de vista estético y de conservación, sino también entendiéndolo como el estudio de la realidad cultural y social de la expresión artística. Así, cabe comprender que cualquier producción y/o interpretación artística se nutre de los acontecimientos de su entorno, apoyándose de las apreciaciones, sensaciones e imaginarios de la ciudad (Díaz, 2021, p.152).

En Amoraditas, hablamos de procesos artísticos y creativos dentro de un contexto social complejo, apostando por el arte "contextual" descrito por Ardene (2006), donde la obra de arte establece una relación directa con la realidad en cuestión, generando casi una co-presencia. Así, esta perspectiva ofrece la posibilidad de subrayar cuestiones que la cotidianidad tiende a censurar u omitir. Por ende, las intervenciones se convierten en verdaderas representaciones de la cultura, contexto social y momento histórico a las que se adscriben.

Del mismo modo, en su vertiente más comunitaria, la iniciativa también bebe del Arte Relacional descrito por Bourriaud (1998), donde se le da una mayor importancia a las relaciones que se establecen entre los participantes del proceso que al objeto artístico en sí mismo. Todas nuestras propuestas de intervención urbana tienen un carácter participativo (Figura 7). Esta aportación por parte de comunidad puede ser total, involucrando a la ciudadanía en todas las partes del proceso; o parcial, tomando decisiones, deseos e información obtenida de los diversos encuentros y reuniones para configurar una propuesta no definitiva a testar y mejorar colectivamente. Así, versamos de arte colaborativo y movimientos socioculturales desde una perspectiva del compromiso social.

La valoración de las intervenciones urbanas, distinta al resto de patrimonios, al abrazar su condición efímera y plantearse la posibilidad de la no preservación, revela la importancia de centrar los esfuerzos en convertir las debilidades del arte urbano en sus fortalezas, entendiendo su deterioro natural como parte de la evolución de ciudades y barrios. Sin embargo, su impronta debe quedar documentada, estableciéndose sistemas de registro que permitan no solo documentar la participación activa, sino también la capacidad de representar vivencias y sentimientos comunitarios (García y Luque, 2021, p.106). La construcción de archivos que incluyan tanto la memoria oral como elementos e hitos de los orígenes y evolución de este tipo de intervenciones resulta esencial para "calibrar su importancia, impacto e influencia en el ámbito cultural y social de las ciudades y establecer vías de diálogo con el campo del patrimonio cultural" (Navarro, 2021, p.123). 


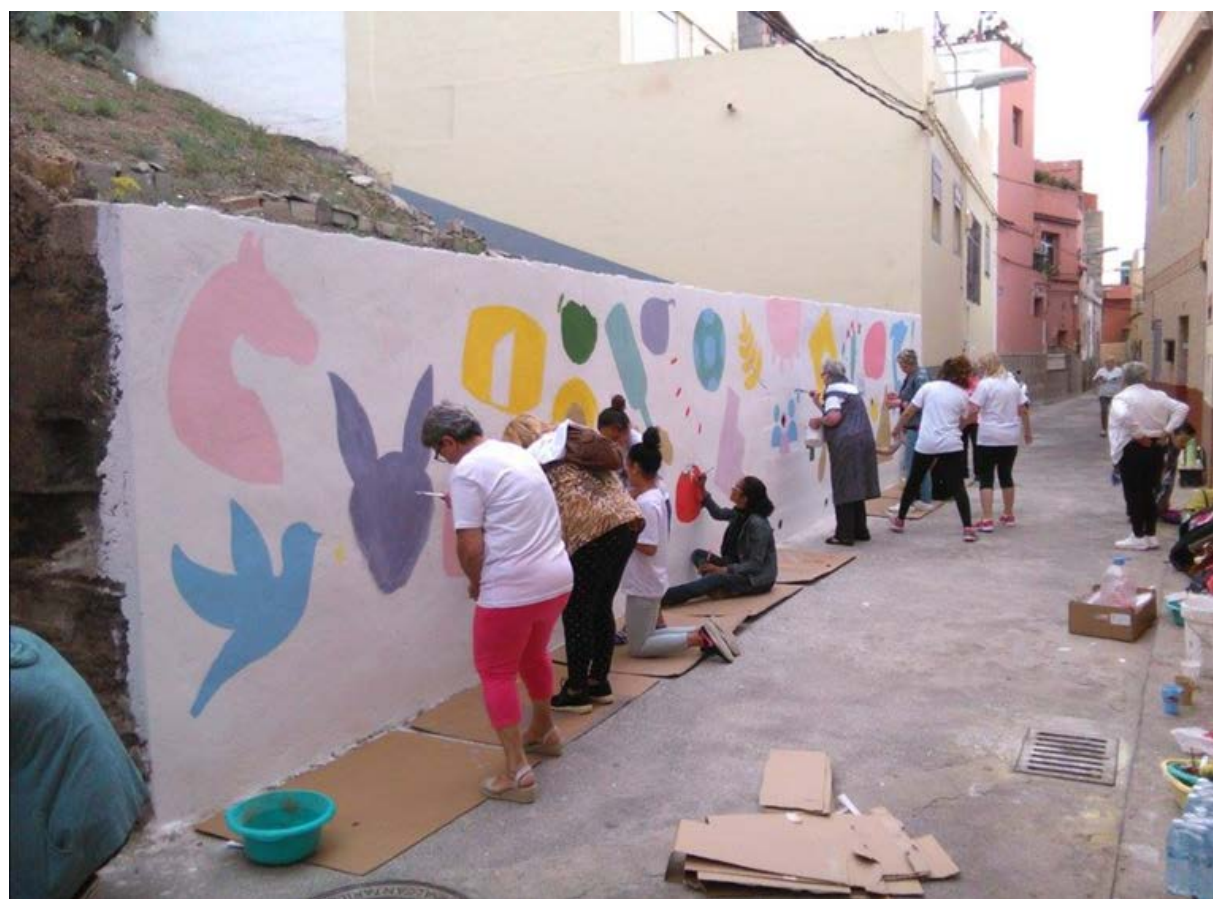

Figura 7. Mural comunitario realizado en el marco de una de las actividades más relevantes del ICI-Taco: el CONvive-Taco. Fuente: Elaboración propia, 2017.

\section{Barrios Artísticos o Distritos Culturales}

Configurando el marco estratégico de la revitalización urbana, los "Barrios Artísticos", como se les designaba tradicionalmente, o los "Distritos Culturales", concepto más actual, actúan como catalizadores del sistema de arte contemporáneo, aunando a artistas e instituciones culturales tales como: talleres, estudios, arte público, museos, galerías de arte o academia. Los ahora llamados Distritos Culturales, pues engloban su especificidad como lugares de alto nivel cultural y son más afines a las consideraciones actuales del rol de las industrias creativas, resultan elementos fundamentales para la configuración de la imagen de la ciudad. Su actual potencialidad trasciende de la conservación patrimonial, configurando generadores de recursos y crecimiento económico que favorecen el desarrollo cultural. (Grupo de Investigación de Arte, Arquitectura y Comunicación en la Ciudad Contemporánea, 2018, p.173-175).

Atendiendo a la definición de Luciana Lazzeretti (2008), los distritos culturales y creativos son lugares de alto nivel cultural y artístico cuyo modelo de estructuración se centra en el desarrollo económico sostenible, basado en el trinomio cultura-economía-sociedad y en el eje recursos-actores-comunidad, que estila a la elevación de las diferencias artísticas, culturales, sociales y medioambientales de las diferentes localidades. Se trata de la conjunción de actores institucionales, económicos y no económicos que a través de la utilización de recursos idiosincrásicos compartidos (artísticos, culturales, sociales, medioambientales), desarrollan un proyecto común, que además posee un carácter económico y resulta casi un proyecto de vida. 
Al analizar dichos distritos se observa la presencia de tres elementos básicos: (1) el alto nivel cultural identificado a través de la idiosincrasia y propios recursos del lugar; (2) la revalorización y mejora económica de los recursos culturales y (3) la localización de recursos y actores de proximidad territorial (Lazzeretti, 2008, p.332). Dentro de los distritos estudiados, el patrimonio y su conservación resulta una de las piedras angulares, al tratarse de elementos culturales claves para la renovación urbana, la revitalización social, la regeneración económica y la promoción cultural. (García, 2021, p.141).

Sin embargo, Amoraditas, a pesar de partir de la centralidad de la Tenerife Design Week 2016 donde existe un enfoque desde lo institucional hacia abajo (top-down), posee una perspectiva alejada de la denominación de distrito cultural, generando un equilibrio de poderes al partir de la comunidad y los recursos existentes (bottom-up). Así, pretende establecer nuevas centralidades, desvinculandose de iniciativas con barrera institucional como el caso del "Distrito de las Artes" en Santa Cruz de Tenerife.

Sin embargo, no se trata de un proceso ajeno a la organización, de hecho, cuenta con las instituciones y trata de congregar a todos los agentes involucrados en el territorio, pero sin esperar por ellos para pasar a la acción. Se trata de una reconciliación entre los organismos y los contextos sociales en las que se inscriben, potenciando la dinamización de la periferia más allá de los ejes territoriales. Partimos de la base de que todos los barrios son culturales, pues los humanos somos intrínsecamente cultura y la ubicación geográfica no debe determinar la facilidad de accesibilidad o derecho a la educación de una comunidad.

Esta tensión entre la alta y la baja cultura dificulta la valorización de culturas no oficiales, como el caso de Las Moraditas como barrio autoconstruido por inmigrantes en los años 50 . Las comunidades periféricas hoy detentan el reto de reconciliar la financiación pública con la ciudadanía a la que le sirve, pero no por ello debe limitar su capacidad de crear, promocionar y consumir arte y cultura. De esta forma, no hablamos de una promoción excesiva del territorio, pues el riesgo de gentrificación puede volverse en contra, sino de una reconciliación tal como la ausencia de jerarquización en los territorios. Un perfecto ejemplo de esto es la ausencia de cartel propio de Las Moraditas, enfatizando su invisibilidad, pero sí la utilización de este territorio, próximo al centro de Santa Cruz, como soporte promocional del cartel del Auditorio de Tenerife, a más de 5 kilómetros del barrio.

\section{HACIA LA ELABORACIÓN DE MARCOS DE TRABAJO TEÓRICOS, INSTRUMENTALES Y OPERATIVOS}

La intervención en diseño para el territorio pone de manifiesto la relevancia de explicitar y compartir los marcos de pensamiento y acción desde los que se parte. En muchas ocasiones, dichos marcos son también puntos de llegada, que solo es posible modelizar una vez sistematizados con una visión retrospectiva. Igualmente, al trabajar conjuntamente con otros perfiles profesionales y áreas de conocimiento que traen sus propios modos de hacer y recursos metodológicos a los procesos comunitarios, encontramos fundamental el establecer glosarios, vocabularios y enfoques comunes, que puedan aunar las diferentes visiones y facilitar su adopción y comunicación entre las partes implicadas. Así, en Amoraditas, se parte de un modelo provisional como hipótesis de trabajo en continua iteración, denominado procediendo (Jiménez-Martínez, 2015), basado en los principios de investigación-acción participativa aplicado al trabajo social comunitario (Marchioni, 1999), comunidades de práctica y aprendizaje 
(Wenger, 2001) y gestión de los bienes comunes (Ostrom, 2000), entre otros. Igualmente, desde la educación superior en diseño y su vinculación con el territorio en que está radicada, está presente el enfoque de aprendizaje-servicio entendido como "una metodología que combina en una sola actividad el aprendizaje de contenidos, competencias y valores con la realización de tareas de servicio a la comunidad, partiendo del principio del apoyo mutuo como mecanismo de progreso personal, económico y social (Puig Rovira, 2009).

Lejos de un recetario, se pretende esbozar aquí el modelo seguido en Amoraditas, de carácter teórico, instrumental y operativo para facilitar la creación de laboratorio vivos y descentralizados de capacitación en I+D+i, para la dinamización de procesos de co-diseño, basados en las demandas de la comunidad local a partir de los recursos territoriales existentes, orientado a la mejora de sus condiciones de vida, con criterios de sostenibilidad (Tabla 1 y Figura 8).

\begin{tabular}{|l|l|}
\hline \multirow{2}{*}{ FASES GENERALES } & MOMENTOS ESPECÍFICOS \\
\hline \multirow{2}{*}{ 1. Definición } & 1.1. Establecimiento del sistema \\
\cline { 2 - 3 } 2. Investigación & 1.2. Creación de la comunidad de diseño \\
\hline \multirow{2}{*}{ 3. Análisis } & 2.1. Conocimiento del contexto \\
\cline { 2 - 3 } & 2.2. Conocimiento de la gente \\
\cline { 2 - 3 } & 3.1. Interpretación y diagnóstico \\
\hline \multirow{2}{*}{ 4. Concepto } & 4.1. Identificación de oportunidades proyectuales \\
\cline { 2 - 3 } & 1.2. Elaboración de un programa de acción integral \\
\hline \multirow{2}{*}{ 5. Proyecto } & 5.1. Implementación \\
\cline { 2 - 3 } & 5.2. Devolución y evaluación comunitaria \\
\hline
\end{tabular}

Tabla 1. Fases generales y momentos específicos del modelo procediendo de diseño. Fuente: Elaboración propia. 


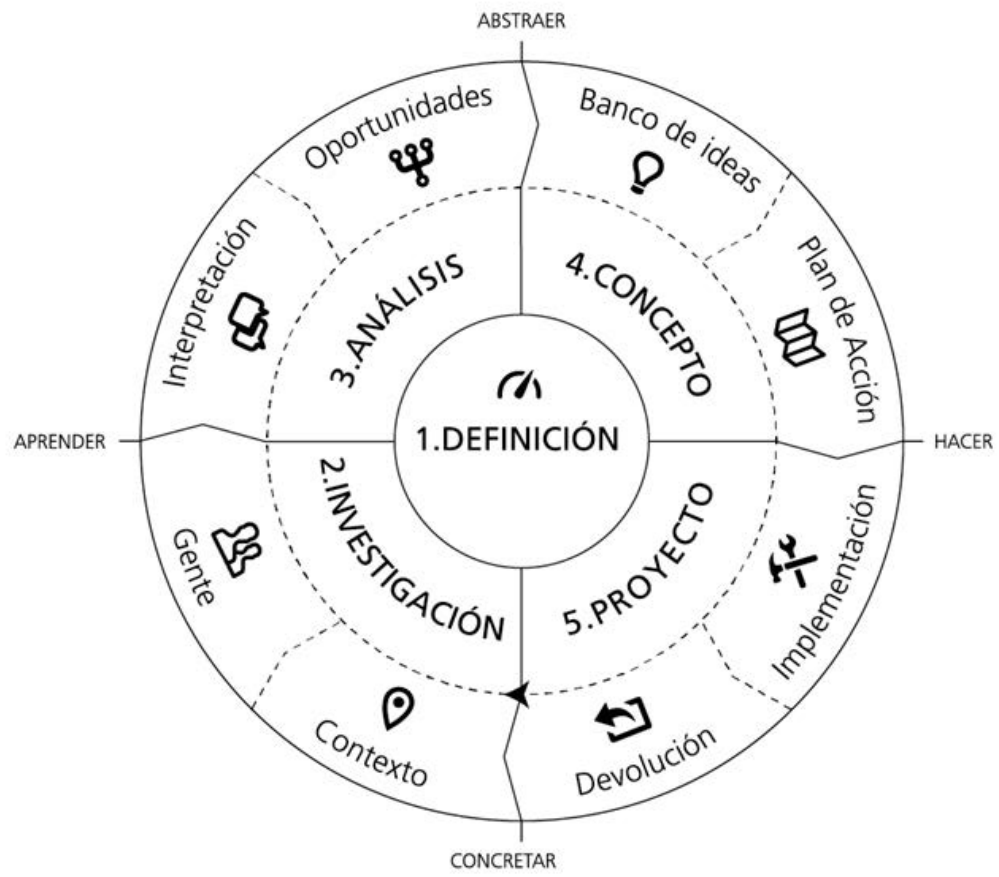

Figura 8. Modelo "procediendo" de diseño aplicado en Amoraditas. Fuente: Jiménez-Martínez, 2015.

\section{CONCLUSIONES}

Amoraditas como iniciativa para la recuperación de la memoria colectiva y la dignificación del de Las Moraditas a través del diseño simboliza un proceso de construcción comunitaria, "un laboratorio de formas vivas que cualquiera puede apropiar" (Bourriaud, 2008). Así, se promociona y paulatinamente se va trascendiendo de la concepción de "él barrio de autoconstrucción colectiva" a "la autoconstrucción colectiva del barrio". Durante su desarrollo:

- se han propiciado lugares de encuentro y conocimiento compartido en el barrio;

- se ha hecho a los vecinos cuestionar su entorno y tratar de idear mecanismos para su mejora, fomentando consecuentemente el sentido de pertenencia;

- se ha conseguido trabajar en equipo para un bien común, eliminando las barreras intergeneracionales e interculturales;

- se ha contribuido a la visibilización y proyección exterior de valores del barrio contra los prejuicios establecidos de esta zona;

- se ha recopilado y difundido nueva información del barrio de Las Moraditas que será preservada; 
- se ha congregado a una serie de entidades que trabajan, de cierta forma, en este barrio y que hasta el momento no habían trabajado conjuntamente;

- se ha empleado el diseño y el arte como catalizadores y elementos vitales del proceso;

- y por último, se ha generado un modelo que puede ser replicado en otras comunidades periféricas con características y objetivos similares.

Este proceso ha contribuido a sensibilizar y educar en materia de diseño no solo a la ciudadanía sino a instituciones y colectivos implicados cuya vinculación con esta disciplina no resulta del todo habitual. Así, han adquirido competencias creativas, convirtiéndose en diseñadores de su propio entorno, apoyándose en el diseñador experto como facilitador del proceso. Si bien hablamos de esta aceptación de papel de esta clase de profesionales, resulta imprescindible señalar los múltiples roles que pueden y son adquiridos por los diseñadores en los procesos comunitarios: desde gestión del diseño, diseño social o diseño o visualización gráfica, hasta gestión y dinamización cultural o incluso un papel más antropológico.

Se evidencia como las metodologías y modos de hacer propios de esta disciplina son capaces de enriquecer procesos comunitarios en gran medida, posicionándose como herramienta de transformación social e impulsora del cambio, siempre y cuando se haga un uso consciente y adecuado de la misma. Toda iniciativa en desarrollo local comunitario implica la inmersión profunda del diseño en el contexto; escenarios complejos, de relaciones cambiantes y resultados con carácter especialmente procesual y preliminar. En estos procesos, se ha demostrado cómo el pensamiento en diseño es efectivo visualizando, prototipando y centrándose en los usuarios. Así, la contribución del diseño no radica tanto en la invención de soluciones, como en facilitar una comprensión compartida de las alternativas de resolución entre los agentes implicados. Una aportación en la que el objetivo del diseño sea la acción coherente entre medios-comunidadfines.

\section{CONTRIBUCIÓN DE AUTORES}

A.M.P., C.J.M. Revisión de literatura (estado del arte): A.M.P., C.J.M. Metodología: C.J.M. Análisis de resultados: A.M.P. Discusión: A.M.P. Conclusiones: C.J.M. Redacción (borrador): A.M.P., C.J.M. Revisiones finales: A.M.P., C.J.M. Diseño del proyecto: A.M.P., C.J.M. 


\section{REFERENCIAS}

Abarca-Álvarez, F. J. y Campos-Sánchez, F. S. (2013). Urbanismos sin lugar: Paisajes participados. Bitácora Urbano-territorial, 22(1), 53-60. https://revistas.unal.edu.co/index.php/bitacora/article/ view/53-60

Ardene, P. Un arte contextual: Creación artística en medio urbano, en situación, de intervención, de participación. Murcia: CENDEAC.

Barrientos, N. (2 de mayo del 2012). ¿Qué es el Placemaking? Plataforma Urbana. https://www. plataformaurbana.cl/ archive/2012/05/02/\%C2\%BFque-es-el-placemaking/

Blanco, P., Carrillo, J. Claramonte, J. y Expósito, M. (2001). Modos de hacer: arte crítico, esfera pública y acción directa. Salamanca: Universidad de Salamanca. https://ciudadcomentada.files. wordpress.com/2015/04/modos-de-hacer.pdf

Bonsiepe, G. (1985). El diseño de la periferia. Debates y experiencias. México: G.Gili.

Bourriaud, N. (2008). Estética relacional. Buenos Aires: Adriana Hidalgo editora.

Díaz, A. (2021). Arte y Patrimonio. Un discurso del tiempoespacio desde lo urbano. Revista PH, 103, 152-154. https://doi. org/10.33349/2021.103

Di Siena, F. (30 de junio de 2015). Diseño Cívico. Urbano Humano. https://urbanohumano.org/blog/2015/06/30/disenocivico/

Di Siena, F. (2016). Introducción. En Diseño Cívico. Diseño, Territorio e Inteligencia colectiva, trabajo colaborativo desarrollado en la Primera edición del curso online de Diseño Cívico entre septiembre de 2015 y febrero de 2016 (pp. 6-8). CivicWise. https://issuu.com/civicwise/docs/publicaci_n_ dise_o_c_vico_sd

Di Siena, F. y Pita, M.J. (2016). Diseño Cívico en 20 puntos. En Diseño Cívico. Diseño, Territorio e Inteligencia colectiva, trabajo colaborativo desarrollado en la Primera edición del curso online de Diseño Cívico entre septiembre de 2015 y febrero de 2016 (pp. 9). CivicWise. https://issuu.com/civicwise/docs/publicaci_n_ dise_o_c_vico_sd 
Fundación "La Caixa”. El Observatorio Social. (s.f.). Proyecto de Intervención Comunitaria Intercultural (ICI). Fundación "La Caixa". https://observatoriosociallacaixa.org/-/proyecto-deintervencion-comunitaria-intercultural-ici

García, J. (2021). El arte urbano como patrimonio cultural, clave en los distritos culturales y creativos. Revista PH, 103, 141-142. https://doi.org/10.33349/2021.103

García, E. y Luque, L. (2021). Dilemas del arte urbano como patrimonio. Revista PH, 103, 106-107. https://doi. org/10.33349/2021.103

Giménez, C., Lobera, J., Mora, T. y Roche, D. (2015). Encuesta 2015 sobre convivencia intercultural en el ámbito local, Informe Territorial de Taco. Obra Social "La Caixa". https:// fundacionlacaixa.org/documents/10280/181359/Encuesta+201 $5+$ sobre+convivencia+intercultural+en+el+\%C3\%A1mbito+local. pdf/af428533-a991-4d61-8f10-e63e6402bfe9

García, G. (2021). Arte urbano: entre la disidencia y la institucionalización. El caso de la ciudad de Valencia. Revista PH, 103, 155-157. https://doi.org/10.33349/2021.103

Greenwood, D., Whyte, W. and Harkavy, I. (1993). Participatory Action Research as a Process and as a Goal. Human Relations, 46(2),175-192.

Grupo de Investigación de Arte, Arquitectura y Comunicación en la Ciudad Contemporánea. (2018). “Barrios Artísticos y Distritos Culturales. Nuevos espacios para la creatividad y la revitalización urbana". Miguel Ángel Chaves Martín, Jesús-Pedro Lorente (Eds). Revista Arte y Ciudad, 13, 171-176. https://doi.org/10.22530/ ayc.2018.N13

Jiménez-Martínez, C. (2015). Cultura del diseño y desarrollo local sostenible. Aportes teóricos, metodológicos y casos prácticos en las Islas Canarias. Servicio de Publicaciones de la Universidad de La Laguna. La Laguna. ISBN: 978-84-16464-45-6. https://shorturl. at/alsDY

Navarro, P. (2021). Construir un patrimonio desde abajo. Revista PH, 103, 122-123. https://doi.org/10.33349/2021.103

Lazzeretti, L. (2008). El distrito cultural. Mediterráneo económico, 13, 327-351. https://publicacionescajamar.es/publicacionesperiodicas/mediterraneo-economico/mediterraneo-economico13-los-distritos-industriales/225 
Lewin, K., Tax, S., Stavenhagen, R., Fals, B., Zamosc, L., Kemmis, S. y Rahman, A. (1946). La investigación-acción participativa. Inicios y desarrollos. Editorial Popular S. A. https://journals.sagepub. com/doi/abs/10.1177/001872679304600203

Manzini, E. (2016). Cuando todos diseñan. Una introducción al diseño para la innovación social. Experimenta Editorial: Madrid.

Marchioni, M. (1999). Comunidad, Participación y Desarrollo. Teoría y metodología de la intervención comunitaria. Madrid: Editorial Popular.

Mau, B. (1998). Manifiesto incompleto para el crecimiento. Conferencia en el Netherlands Design Institute. Traducción de Jaume Pujagut.

Meroni, A.; Selloni, D. and Rossi, M. (2018). Massive Codesign. A Proposal for a Collaborative Design Framework. https:// www.researchgate.net/publication/337286186_MASSIVE_ CODESIGN_A_Proposal_for_a_Collaborative_Design_Framework

Ministerio de Educación y Formación Profesional. (s.f.). Cómo hacer patrimonios comunes en el rural. La aventura de aprender: proyectos colaborativos. http://laaventuradeaprender.intef.es/ guias/-como-hacer-patrimonios-comunes-

Organización de las Naciones Unidas para la Educación, la Ciencia y la Cultura. (s.f.). ¿Qué es el patrimonio cultural inmaterial? UNESCO. https://ich.unesco.org/es/qu-es-el-patrimonioinmaterial-00003

Ostrom, E. (2000). Diseños complejos para manejos complejos. Gaceta Ecológica, 54, 43-58.

Participatory Research Cluster, Institute of Development Studies, supported by the Swiss Agency of Development and Cooperation. (n.d.) Participatory Action Research. https://www. participatorymethods.org/glossary/participatory-action-research

Pilay, L. y Neves, M. (2020). Diseño para la valoración del patrimonio cultural-gráfico de la ciudad. Gráfica, 8(15) 55-61. https://doi.org/10.5565/rev/grafica.171

Project for Public Spaces (2017). What is placemaking? https:// www.pps.org/article/what-is-placemaking

Puig Rovira, J.M. (Coord). (2009). Aprendizaje servicio (ApS): educación y compromiso cívico. Barcelona: GRAO. 
Tenerife Design Week. Connecting creative communities. (s.f.). Exposición: "Living Design Lab". https://tenerifedesignweek. com/living-design-lab-2/

Torres-Tovar, A. (2019). Movilidad, espacio público y diseño participativo. Bitácora, 29, 7-8. https://doi.org/10.15446/ bitacora.v29n3.82468

Universitat Oberta de Catalunya. (s.f.). Design Toolkit:

Codiseño, ¿qué es? http://design-toolkit.uoc.edu/es/codiseno/

Universitat Oberta de Catalunya. (s.f.). Design Toolkit: Diseño centrado en las personas, ¿qué es? http://design-toolkit.uoc. edu/es/codiseno/

Wenger, E. (2001). Comunidades de práctica: aprendizaje, significado e identidad. Barcelona: Paidós.

Zapata, V., Mesa, A. y Fonte, M. (2020). Procesos comunitarios como vías de inclusión social: La participación de las personas inmigrantes en el proyecto ICI Taco de Tenerife. Cuadernos Manuel Giménez Abad, (M 7 - marzo 2020), 58-69. https:// dialnet.unirioja.es/servlet/articulo?codigo $=7278515$

1. Puesto que todo el mundo posee la capacidad natural de diseñar, Manzini distingue entre diseñadores difusos, cualquier individuo, y diseñadores expertos, personas formadas en diseño o profesionales de esta disciplina. La interacción de ambos contribuye a alcanzar objetivos más complejos e impulsar cambios sociales significativos (Manzini, 2015, p.47). 